Bedeutung bei. Außerdem erscheinen die Parlamente zu sehr als einheitliche Akteure. Die funktionslogische Trennung von Regierung und Opposition mit ihren unterschiedlichen Anreizen zu Mitwirkung, Kontrolle oder wohlwollender Begleitung des Regierungshandelns, die sich aus der jeweils spezifischen dynamischen Beziehung zwischen Exekutive und Legislative eröffnet ${ }^{4}$, bleibt weitgehend unbeachtet. Wünschenswert wären auch mehr Präzision und Tiefe bei Begriffsbestimmungen der unterschiedlichen Facetten von Kontrolle und Mitwirkung gewesen. ${ }^{5}$

Insgesamt gelingt Ondarza in seiner Dissertation aber ein guter exemplarischer, empirisch reichhaltiger und übersichtlich strukturierter Einblick in die Entscheidungsverfahren zur Entsendung von Streitkräften in multilaterale Missionen. Der Vergleich der Einbindungsmuster von fünf nationalen Parlamenten enthüllt jedoch nur den offen sichtbaren Teil ihrer Rolle als Träger der demokratischen Legitimation dieser sensiblen sicherheitspolitischen Entscheidungen. Die darunter liegenden Schichten informeller Kontroll- und Mitwirkungsmöglichkeiten werden zu wenig freigelegt.

Auf diese Weise bestätigt sich die im Titel aufgeworfene Frage als selbsterfüllende Prophezeiung: Parlamente blieben somit in der Sicherheitspolitik trotz allmählicher Verbesserung vorerst doch „bloße Notare anderweitig getroffener Entscheidungen “6 und damit „Legitimatoren ohne (großen) Einfluss“. Ein differenzierteres Bild würde entstehen, wenn man die parlamentarische Funktionslogik von Regierung und Opposition systematisch betrachten - und damit informelle Kanäle und Strukturen der parlamentarischen Mitwirkung aufspüren würde.

René Lüddecke

4 Vgl. unter anderem Everhard Holtmann / Werner J. Patzelt (Hrsg.), Kampf der Gewalten? Parlamentarische Regierungskontrolle - gouvernementale Parlamentskontrolle, Wiesbaden 2004.

5 Siehe bereits bei Winfried Steffani, Gewaltenteilung und Parteien im Wandel, Wiesbaden 1997; Richard G. Mulgan, Holding Power to Account. Accountability in Modern Democracies, Basingstoke 2003.

6 Jean Blondel, Comparative Legislatures, Englewood Cliffs 1973, S. 22.

\title{
Europapolitische Mitwirkung des Bundestags - präzise Beschreibung mit konzeptionellen Schwächen
}

Schulz, Fabian: Die Mitwirkung des Deutschen Bundestags in europäischen Angelegenheiten. Politikgestaltung im Spannungsfeld von Europäisierung und Entparlamentarisierung, Waxmann Verlag, Münster 2011, 312 Seiten, €29,90.

Wie gestalten sich die Beteiligungsrechte des Deutschen Bundestags in europäischen Angelegenheiten, und auf welchen Wegen findet parlamentarische Mitwirkung tatsächlich statt? Diese Fragen bilden den Ausgangspunkt der von Fabian Schulz vorgelegten Arbeit zur europapolitischen Mitwirkung des Bundestags. Der analytische Fokus der erklärtermaßen deskriptiven Studie liegt auf den "tatsächlich praktizierten Mitwirkungswegen“ (S. 12). Diese können, wie Schulz hervorhebt, sowohl auf formalen als auch auf informalen Beteili- 
gungsmöglichkeiten basieren und inzwischen auch die unmittelbare Mitwirkung der Bundestagsabgeordneten auf europäischer Ebene einschließen. Mit der qualitativen Analyse der Praxis leistet Schulz einen entscheidenden Beitrag zur Forschung über die Rolle nationaler Parlamente im europäischen Mehrebenensystem. Er knüpft dabei an die bekannten Einsichten an, dass sich das Ausmaß und der Effekt parlamentarischer Mitwirkung in EU-Angelegenheiten mit Blick auf die formalen Beteiligungsrechte allein nicht hinreichend erfassen lässt und die an Bedeutung gewinnenden direkten Mitwirkungswege auf der Gemeinschaftsebene weiterer Erforschung bedürfen.

Schulz hat 27 leitfadengestützte Experteninterviews mit Bundestagsabgeordneten, Mitarbeitern verschiedener parteipolitischer Zugehörigkeit und Angehörigen der Bundestagsverwaltung geführt. Ergänzt um Primärquellen und die einschlägige Literatur hat er so eine sehr fundierte Datenbasis geschaffen, die ihm erlaubt, in anvisierter Weise die „Komplexität so weit wie möglich zu erfassen und in detaillierter, oftmals kleinschrittiger Herangehensweise alle relevanten Aspekte des Themas zu bearbeiten“ (S. 266).

Schulz' empirische Analyse zeigt, dass die in Übereinstimmung mit bestehenden Studien als „parlamentsfreundlich“ (S. 47) gewerteten formalen Verfahren parlamentarischer Mitwirkung in der Praxis nur sehr verhalten genutzt werden. Auch Optionen zur direkten Beteiligung auf Gemeinschaftsebene werden von den Parlamentariern kaum aufgegriffen. Weitaus wichtiger sind die informalen Verfahren innerstaatlicher parlamentarischer Mitwirkung: Die Mehrheitsfraktionen beschreiten insbesondere den Weg der internen, leisen Mitwirkung, zum Beispiel durch die Beeinflussung von Regierungsmitgliedern im Rahmen von Arbeitsgruppen der Fraktion. Dieser ,intra-party-mode' kann gemäß Schulz insbesondere deshalb seine Wirkung entfalten, weil die Regierung auch in europäischen Angelegenheiten grundlegende fraktionsinterne Konflikte vermeiden und sich der dauerhaften Akzeptanz ihrer parlamentarischen Basis versichern muss. Doch auch Schulz' Analyse bestätigt bekannte Dilemmata der Mehrebenenpolitik. Die Mehrheit kann der Regierung nicht zu stark die Hände binden, sofern sie deren Verhandlungschancen auf europäischer Ebene nicht schmälern will. Insofern wird die grundlegend dominante Position der Regierung im Rahmen der informalen fraktionsinternen Mitwirkung nicht aufgehoben. Die Mehrheitsfraktionen übernehmen deshalb, wie Schulz unter Rückgriff auf bestehende Studien präzisiert, vorrangig die Funktion der ,monitoring scrutiny'. Sie nutzten fraktionsinterne Kommunikationskanäle, um Informationen über Regierungsaktivitäten auf europäischer Ebene zu verlangen und das Regierungshandeln frühzeitig im Rahmen der Möglichkeiten an ihre Interessen zurückzubinden.

Den Oppositionsfraktionen bietet sich zur Einflussnahme vornehmlich die Nutzung der Öffentlichkeit an. Aufgrund der hinlänglich bekannten Probleme (zum Beispiel mangelndes öffentliches Interesse an europäischer Politik) erweist sich dieser Mitwirkungsweg jedoch kaum als fruchtbar und konnte, wie Schulz demonstriert, „,bislang kaum zu erkennbaren Ergebnissen“ (S. 137 f.) führen. Die in parlamentarischen Systemen vorrangig der Opposition zufallende ,political scrutiny', also die offene Kritik des Regierungshandelns und das Aufzeigen politischer Alternativen, bleibt damit bezüglich europäischer Angelegenheiten recht schwach. Auch bestehende Kompensationsmöglichkeiten - etwa die Einflussnahme durch die Nutzung direkter Kontakte auf der Gemeinschaftsebene oder die Instrumentalisierung des Bundesrates - werden von der Opposition nicht wahrgenommen. Der tatsächliche Einfluss der innerstaatlichen parlamentarischen Mitwirkung fällt vor diesem Hintergrund insgesamt moderat aus. Angesichts dieser Befunde beendet Schulz seine Studie mit praxisorientierten Reformvorschlägen zu ihrer Stärkung. 
Insgesamt zeichnet Schulz ein sehr differenziertes und praxisnahes Gesamtbild der parlamentarischen Beteiligungswege in europäischen Angelegenheiten. Danach sind die formalen Verfahren mit dem durch Gewaltenverschränkung geprägten politischen System der Bundesrepublik wenig kompatibel und insofern in der Praxis gegenüber den spezifischen informalen Mitwirkungswegen von Mehrheits- und Oppositionsfraktionen kaum von Bedeutung.

Ein Schwachpunkt der Studie liegt in der speziell zu Beginn der Arbeit recht losen Anbindung an bestehende Forschungsergebnisse. Analysen der praktischen Mitwirkung des Deutschen Bundestags in europäischen Angelegenheiten, die später durchaus Erwähnung finden (zum Beispiel S. 51), werden einleitend kaum diskutiert. Die vierseitige Darlegung des Forschungsstandes erscheint somit als Pflichtübung. Sie wird weder zur Präzisierung zentraler Konzepte genutzt (Mitwirkung), noch dient sie der Generierung plausibler Erwartungen hinsichtlich der akteursspezifischen Ziele und Mechanismen der Mitwirkung sowie der entsprechenden Instrumente. Hier hätte die Studie durch eine einleitende, von Beginn an entlang des neuen Dualismus strukturierte Diskussion möglicher Mitwirkungswege an Stringenz gewonnen. So mag es zum Beispiel angesichts vielfältiger Ausführungen über die für parlamentarische Systeme typische Verzahnung von Regierung und Parlamentsmehrheit nicht gerade überraschen, dass die Einflussnahme in europäischen Angelegenheiten seitens der Mehrheitsfraktionen im ,intra-party-mode' erfolgt, zumal der Autor selbst betont, dass das „Konzept einer informalen Mitsteuerung allgemein anerkannt ist“ (S. 163). Ebenso verwundert es angesichts teilweise auch genannter Ausführungen von Arthur Benz sowie Katrin Auel kaum, dass der Opposition zur Mitwirkung trotz aller damit verbundenen Schwierigkeiten in erster Linie der Weg der öffentlichen Kritik des Regierungshandelns offen steht. Schulz' Andeutung, dass bislang keine „klare[n] Ideen - Konzepte - davon bestünden, wie und auf welchen Wegen der Bundestag in europäischen Angelegenheiten mitwirkt" (S. 19), ist in Anbetracht dessen kritisch zu hinterfragen.

Ungeachtet dieser Kritik ist die Studie insgesamt lesenswert. Sie liefert eine umfangreiche Deskription, die an Tiefenschärfe und Aktualität kaum Wünsche offen lässt. Damit leistet Schulz einen wichtigen Beitrag zur empirischen Analyse der tatsächlichen Mitwirkung des Deutschen Bundestags im europäischen Mehrebenensystem.

Lisa Helene Anders

\section{Europarecht - grundlegende Kommentierung und engagierte Positionen}

Schwarze, Jürgen (Hrsg.): EU-Kommentar, 3. Auflage, Nomos Verlagsgesellschaft, Helbing Lichtenhahn Verlag, facultas.wuv Verlag, Baden-Baden / Basel / Wien 2012, 3019 Seiten, $€ 225$,-

Schwarze, Jürgen: Europarecht, Strukturen, Dimensionen und Wandlungen des Rechts der Europäischen Union, Nomos Verlagsgesellschaft, Baden-Baden 2012, 1151 Seiten, € 128,-.

Einen deutlichen Gewinn an Demokratie brachte der EU Ende 2009 der Reformvertrag von Lissabon. Das Europäische Parlament - das Bundesverfassungsgericht verneinte im Lissabon-Urteil vom 30. Juni 2009 abschätzig die Qualität einer Volksvertretung und stellte es 